\title{
Electronic Transport Properties of Hole-Doped $\mathrm{Sr}_{1+x} \mathrm{La}_{1-x} \mathrm{CrO}_{4+\delta}$
}

\author{
Takahisa OMATA, Takashi KAWANO, Hiroyuki IKAWA, Tadashi SASAMOTO, Hideo HOSONO*, \\ Hiroshi MIZOGUCHI** and Hiroshi KAWAZOE** \\ Department of Chemical Technology, Kanagawa Institute of Technology, 1030, Shimo-ogino, Atsugi-shi 243-02 \\ *Institute for Molecular Science, 38, Saigo-naka, Myodaiji, Okazaki-shi 444 \\ **Research Laboratory of Engineering Materials, Tokyo Institute of Technology, 4259, Nagatsuta, Midori-ku, Yokohama-shi

\author{
ホールドープした $\mathrm{Sr}_{1+x} \mathrm{La}_{1-x} \mathrm{CrO}_{4+\delta}$ の電子伝導性

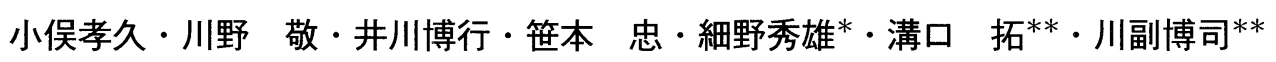 \\ 神奈川工科大学工業化学工学科, 243-02 厚木市下荻野 1030 \\ *分子科学研究所, 444 岡崎市明大寺字西郷中 38 \\ **東京工業大学工業材料研究所, 226 横浜市緑区長津田 4259
}

[Received May 11, 1995; Accepted August 24, 1995]

\begin{abstract}
Polycrystalline bodies of $\mathrm{Sr}_{1+x} \mathrm{La}_{1-x} \mathrm{CrO}_{4+\delta} \quad(0 \leq x$ $\leq 0.3)$ with a $\mathrm{K}_{2} \mathrm{NiF}_{4}$ structure were prepared and the temperature dependence of the electrical conductivity and Seebeck voltage at room temperature were measured. The temperature dependence of conductivity followed a functional form of $\exp \left[\left(-T_{0} / T\right)^{\alpha}\right](\alpha=1 / 3$ or $1 / 4)$, which was characteristic of two- or three-dimensional variable-range hopping (VRH) model. The hopping conductivity showed that the crystal was nonmetallic because the electronic states at the Fermi energy were localized. Values of $T_{0}$ in the three-dimensional VRH regime were in the range of $1-5 \times 10^{8} \mathrm{~K}$. This was larger by two orders of magnitude than reported values in $\mathrm{La}_{2} \mathrm{CuO}_{4}$ which was insulating between room temperature and $2 \mathrm{~K}$. Based on the VRH regime, the larger $T_{0}$ values were considered to be caused by the smaller localization radius of impurity states. All the samples were demonstrated to have a positive Seebeck coefficient. Further, the coefficient decreased with increasing doping level. The composition dependence of the Seebeck coefficient was discussed in relation to the changes with compositions in $T_{0}$ and the density of states in the vicinity of the Fermi energy.
\end{abstract}

Key-words : $\mathrm{Sr}_{1+x} \mathrm{La}_{1-x} \mathrm{Cr} \mathrm{O}_{4+\delta}, \mathrm{K}_{2} \mathrm{NiF}_{4}$ structure, Electrical conductivity, Seebeck coefficient, Variable-range hopping model

1. Introduction

The metal-insulator (MI) transition of 3d transition metallic (TM) complex-oxides including high$T_{\mathrm{c}}$ superconductors has been investigated for complex-oxides with perovskite and related structures. -Much attention, however, has been paid to the metallic and the superconducting states of those oxides. It is considered that investigations on the insulator side of the MI transition is important to find a clue to the riddle of the MI transition. Recently, there are considerable studies on non-metallic states of cuprates. ${ }^{1)-6)}$ In these studies, electrical conductivities of non-metallic and semiconducting states have been well described on the basis of the variablerange hopping (VRH) model. ${ }^{7), 8)}$ In that description the conduction path is assumed to be three-dimensional despite that these crystals have a two-dimension-like character. A large localization length of the impurity states in $\mathrm{La}_{2} \mathrm{CuO}_{4}$ has been reported ${ }^{2), 3)}$ compared with those of amorphous semiconductors, in which the conduction mechanism is well described by a VRH model.

It is expected that further extensions of comparative studies on insulating states of different $3 \mathrm{~d}$ TM complex-oxides may give us some clues to understand the mechanism of the MI transition. In earlier papers, we reported physical properties and the electronic structure of hole-doped $\mathrm{Sr}_{1+x} \mathrm{La}_{1-x} \mathrm{FeO}_{4} \cdot{ }^{9)-11)}$ The results have been analyzed on the basis of a small polaron theory. ${ }^{12)}$ In the present work, it has been studied that electronic transport properties of hole-doped $\mathrm{Sr}_{1+x} \mathrm{La}_{1-x} \mathrm{CrO}_{4+\delta}$ with a $\mathrm{K}_{2} \mathrm{NiF}_{4}$ structure. ${ }^{13)}$ The results obtained are analyzed on the basis of the $\mathrm{VRH}$ regime. The difference between the present system and those cuprates is discussed in respect to characteristics of the impurity states such as a localization length and density of states in the vicinity of the Fermi energy.

\section{Experimental}

Polycrystalline $\mathrm{Sr}_{1+x} \mathrm{La}_{1-x} \mathrm{CrO}_{4+\delta}(0 \leq x \leq 0.3)$ samples were prepared by the following procedures. The powdered raw materials of $\mathrm{SrCO}_{3}(99.9 \%), \mathrm{La}_{2} \mathrm{O}_{3}$ (99.9\%) and $\mathrm{Cr}_{2} \mathrm{O}_{3}(99.9 \%)$ were thoroughly mixed with ethanol by using an agate mortar, and those mixtures were calcined at $1300^{\circ} \mathrm{C}$ for $12 \mathrm{~h}$ under $\mathrm{N}_{2}$ flow. $\mathrm{La}_{2} \mathrm{O}_{3}$ was fired at $1000^{\circ} \mathrm{C}$ for $12 \mathrm{~h}$ in air just before weighing to avoid moisture adsorption. The calcined powder was mixed again by using a planetary mill (Fritsch, pulverisette 5) and was pressed into 10- or 16 -mm-diameter disks under $100 \mathrm{MPa}$. The disks were subsequently sintered at $1500^{\circ} \mathrm{C}$ for $24 \mathrm{~h}$ under $\mathrm{N}_{2}$ flow. Crystalline phases in these sintered samples were identified by an X-ray powder diffraction 
method (Rigaku, RINT2500, $\mathrm{Cu}$ K $\alpha$ radiation). Lattice parameters were calculated by using a leastsquare procedure from relevant diffraction peaks. A high-purity silicon powder was mixed with a sample as an internal standard. The concentration of constituent cations were determined by ICP (inductively coupled plasma) atomic-emission spectrometry (Shimadzu, ICPS-5000). It is known that oxygen nonstoichiometry is observed usually in $3 \mathrm{~d}$ TM oxides, but in this study values of $\delta$ in $\mathrm{Sr}_{1+x} \mathrm{La}_{1-x}$ $\mathrm{CrO}_{4+\delta}$ were not determined, because the controlled valence charge by Sr-substitution is considered to be larger than that comes from oxygen nonstoichiometry.

The dc electrical conductivity of a sample was measured by a standard four-probe technique in a temperature range 77 to $300 \mathrm{~K}$. A rectangularly shaped sample was cut from a sintered disk. Au electrodes were sputtered on surfaces of the sample and those were connected to $\mathrm{Cu}$ wires. The minimum reliable conductivity was approximately $1 \times 10^{-4}$ $\Omega^{-1} \cdot \mathrm{m}^{-1}$ in the present system. Seebeck coefficients were measured at a base temperature of $297 \mathrm{~K}$, and temperature difference of 4-7 $\mathrm{K}$ was set between the two ends of each sample. Two pairs of thin copperconstantan thermocouples were attached at the two ends. The voltage developed across the copper wires of two thermocouples, which is the Seebeck voltage of the sample, was measured up to the order of 0.1 $\mu V$ with a digital voltmeter.

\subsection{Crystal structure}

\section{Results}

Figure 1 shows X-ray diffraction patterns of $\mathrm{Sr}_{1+x}$ $\mathrm{La}_{1-x} \mathrm{CrO}_{4+\delta}$ with $x=0$ and 0.3 . All the diffraction peaks of the sample with $x=0$ were indexed as those of tetragonal $\mathrm{K}_{2} \mathrm{NiF}_{4}$ with a space group of $I 4 / \mathrm{mmm}$. The diffraction patterns of specimens with $x=0.1$ and 0.2 were almost identical with the case of the sample with $x=0$. In the case of the sample with $x=0.3$, weak diffraction peaks due to $\mathrm{La}_{2} \mathrm{O}_{3}$ and $\mathrm{Sr}_{2} \mathrm{CrO}_{4}$ were observed. Lattice parameters are summarized in Table 1 along analytical compositions by ICP analysis. Upon doping, the length of $a$-axis was shortened while that of $c$-axis was stretched. This shows that the substitution of La with Sr progressed up to $x=0.3$. Apparent densities of the sintered disks were $67-89 \%$ of the theoretical densities (Table 1 ).

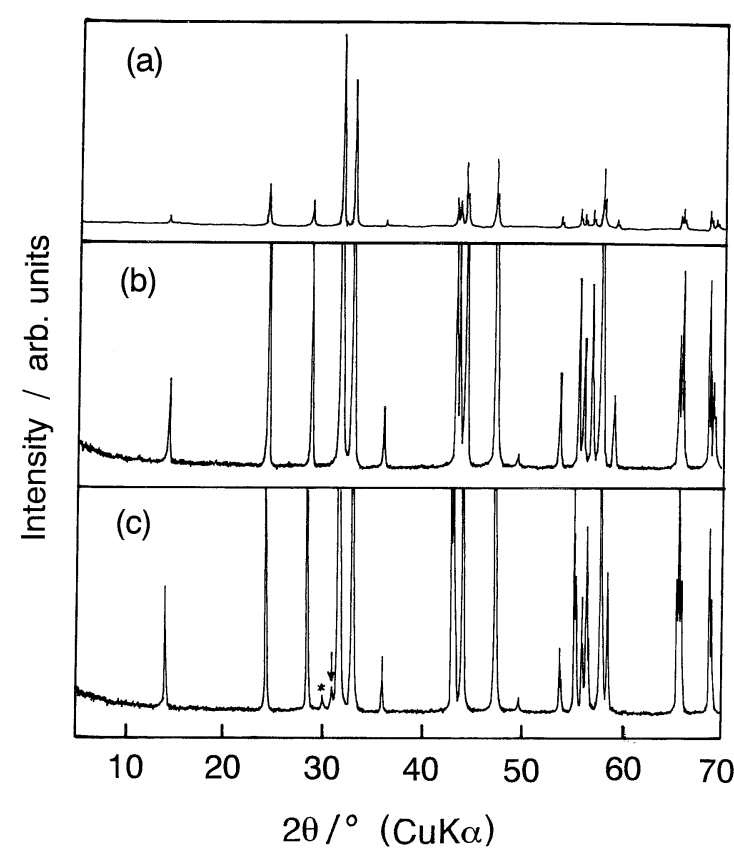

Fig. 1. Powder X-ray diffraction patterns of sintered $\mathrm{Sr}_{1+x} \mathrm{La}_{1-x}$ $\mathrm{CrO}_{4+\delta}$.

(a) $x=0$, (b) magnified pattern of (a) and (c) $x=0.3$. The diffraction peaks marked by an asterisk and an arrow are due to $\mathrm{La}_{2} \mathrm{O}_{3}$ and $\mathrm{Sr}_{2} \mathrm{CrO}_{4}$, respectively.

In the following discussion, the effects of such low densities are disregarded, because the electrical conductivity is considered to be insensitive to the density of the samples.

\subsection{Electronic transport properties}

Figure 2 shows simple Arrhenius plots of electrical conductivity of $\mathrm{Sr}_{1+x} \mathrm{La}_{1-x} \mathrm{CrO}_{4+\delta}$. The conductivity is thermally activated evidently, however, the activation energy shows temperature dependence. The logarithm of the conductivity is plotted against $T^{-1 / 4}$ in Fig. 3 to illustrate the agreement of the result with the variable-range hopping (VRH) law. Solid lines in the figure are least-square fits to a following equation:

$$
\ln \sigma=\ln \sigma_{0}-\left(\frac{T_{0}}{T}\right)^{1 / 4}
$$

Table 2 shows $\sigma_{0}, T_{0}$ and $r$, which is a correlation coefficient between $\ln \sigma$ and $T^{-1 / 4}$, obtained from the fits. The correlation factors were found to be $r>0.9997$. Because of the close values of $r$ to 1 , the

Table 1. Lattice Parameters, Cell Volume, and the Analytical Composition of $\mathrm{Sr}_{1+x} \mathrm{La}_{1-x} \mathrm{CrO}_{4+\delta}$

\begin{tabular}{cccccc}
\hline \multicolumn{3}{c}{$\begin{array}{c}\text { Length of the individual axis } \\
a_{0} / \mathrm{nm}\end{array}$} & $\begin{array}{c}\text { Cell volume } \\
/ \mathrm{nm}^{3}\end{array}$ & Analytical composition & $\begin{array}{c}\text { Relative density } \\
\%\end{array}$ \\
\hline 0 & $0.38605(2)^{*}$ & $1.2492(1)$ & $0.18617(2)$ & $\mathrm{La}_{0.93} \mathrm{Sr}_{0.98} \mathrm{CrO}_{4+\delta}$ & 67 \\
0.1 & $0.38511(2)$ & $1.2510(1)$ & $0.18561(3)$ & $\mathrm{La}_{1.02} \mathrm{Sr}_{1.31} \mathrm{CrO}_{4+\delta}$ & 74 \\
0.2 & $0.38419(2)$ & $1.2533(1)$ & $0.18499(3)$ & $\mathrm{La}_{0.74} \mathrm{Sr}_{1.17} \mathrm{CrO}_{4+\delta}$ & 72 \\
0.3 & $0.38338(2)$ & $1.2554(1)$ & $0.18451(2)$ & $\mathrm{La}_{0.63} \mathrm{Sr}_{1.29} \mathrm{CrO}_{4+\delta}$ & 89 \\
\hline
\end{tabular}

*numbers in parentheses show estimated standard deviations in the last digits. 


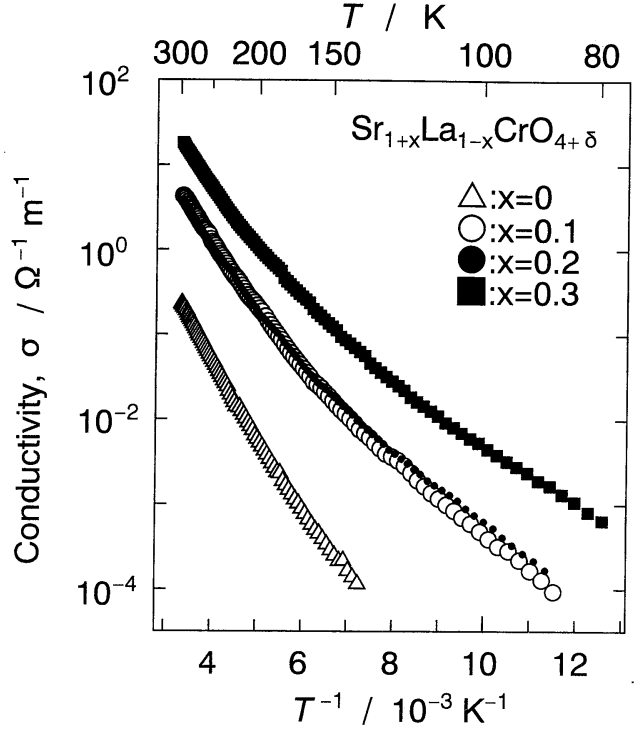

Fig. 2. Reciprocal temperature dependence of the electrical conductivity of $\mathrm{Sr}_{1+x} \mathrm{La}_{1-x} \mathrm{CrO}_{4+\delta}$.

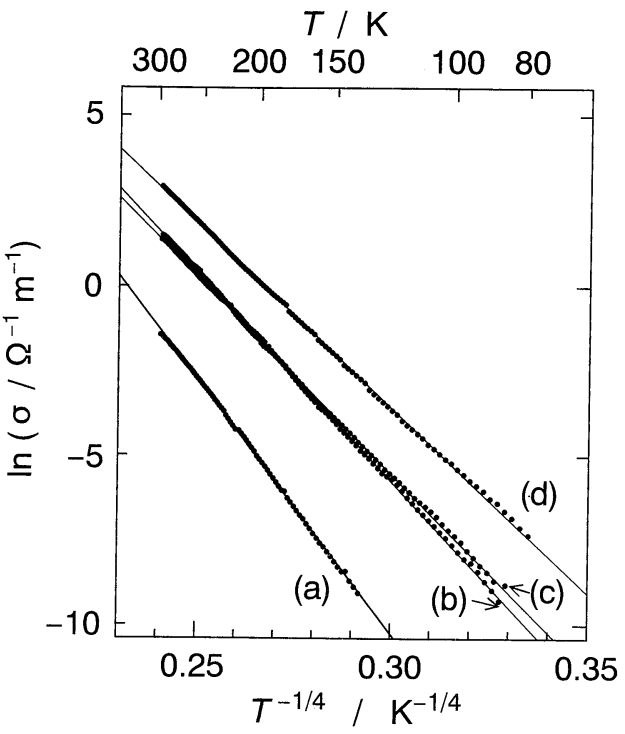

Fig. 3. Electrical conductivity vs. $T^{-1 / 4}$ for the samples of $\mathrm{Sr}_{1+x}$ $\mathrm{La}_{1-x} \mathrm{CrO}_{4+\delta}$.

(a) $x=0$, (b) $x=0.1$, (c) $x=0.2$ and (d) $x=0.3$.

Table 2. Fitting Parameters for $\mathrm{Sr}_{1+x} \mathrm{La}_{1-x} \mathrm{CrO}_{4+\delta}$ of the Eq. (1) and Seebeck Coefficients at Room Temperature

\begin{tabular}{ccccccc}
\hline $\mathrm{x}$ & $\sigma_{0} / \Omega^{-1} \mathrm{~m}^{-1}$ & $T_{0}(\mathrm{x}) / \mathrm{K}$ & $\mathrm{r}$ & $\mathrm{S}(\mathrm{x}) / \mu \mathrm{V} \mathrm{K}^{-1}$ & $T_{0}(\mathrm{x}) / T_{0}(0)$ & $\mathrm{S}(\mathrm{x}) / \mathrm{S}(0)$ \\
\hline 0 & $1.3 \times 10^{11}$ & $5.06 \times 10^{8}$ & 0.9997 & 343 & 1 & 1 \\
0.1 & $3.3 \times 10^{9}$ & $2.27 \times 10^{8}$ & 0.9999 & 178 & 0.45 & 0.52 \\
0.2 & $5.2 \times 10^{8}$ & $1.78 \times 10^{8}$ & 0.9998 & 122 & 0.35 & 0.36 \\
0.3 & $4.0 \times 10^{8}$ & $1.40 \times 10^{8}$ & 0.9998 & 89 & 0.28 & 0.26 \\
\hline
\end{tabular}

logarithm of conductivity shows a well-defined $T^{-1 / 4}$ dependence over whole temperature range. Further, reasonable fits were obtained for the case of plots of $\ln \sigma_{0}$ vs. $T^{-1 / 3}$ with $r$ values of $0.9995-0.9998$.

Table 2 shows positive Seebeck coefficients of each sample and the values decrease with increasing doping level.

\section{Discussion}

\subsection{Electrical conductivity}

The temperature dependence of electrical conductivity is well described by Eq. (1). This shows that the conduction mechanism is represented by the VRH model. ${ }^{7), 8)}$ The electrical conductivity for hopping models has been calculated for a number of different cases. Mott and Davis ${ }^{7)}$ and Shklovskii and Efros $^{8)}$ derived an equation given by

$$
\sigma=\sigma_{0} \exp \left[-\left(\frac{T_{0}}{T}\right)^{\alpha}\right]
$$

where $T_{0}$ is a characteristic temperature, which will be discussed later, and

$$
\alpha=\frac{n+1}{n+D+1}
$$

$D$ defines the dimensionality of the hopping process, and $n$ describes the energy dependence of the densi- ty of states (DOS) in the vicinity of the Fermi energy.

For the Mott-Davis VRH case $(n=0), \alpha$ becomes $1 / 3$ and $1 / 4$ in two and three dimensional conduction, respectively. For the case of Shklovskii and Efros, $n$ is 1 in the two dimensional case, whereas $n=2$ in the three dimensional conduction. This leads to the same exponent $\alpha=1 / 2$ for two and three dimensions in Eq. (3).

The observed conductivity was fitted to the Eq. (2) with $\alpha=1 / 2,1 / 3$ and $1 / 4$, and obtained reasonable fits in cases of $\alpha=1 / 3$ and $1 / 4$. Thus it is concluded that the conduction behavior in this system is well explained by Mott-Davis VRH. Although the best fit is obtained in the case of $\alpha=1 / 4$, it is difficult to distinguish whether the hopping process occurs in three or two dimensions. Wider temperature dependence measurements will be needed to distinguish the dimensions of the hopping process. In the following discussion, we prefer the three-dimensional VRH regime to compare with the result of the analysis based on the three-dimensional VRH model in $\mathrm{La}_{2} \mathrm{CuO}_{4}$ system.

The temperature $T_{0}$ in Eq. (2) is given by Mott and Davis ${ }^{7)}$ as

$$
T_{0}=B_{0} \beta^{3} / k N\left(E_{\mathrm{F}}\right)
$$

where $B_{0}$ is a dimensionless constant, $\beta$ is the 
coefficient of exponential decay of the wave-function of localized states, i.e., $1 / \beta$ expresses the radius of the localized states. And $N\left(E_{\mathrm{F}}\right)$ is the DOS at the Fermi level and $k$ is the Boltzman constant in Eq. (4). Table 2 shows that $T_{0}$ decreases with increasing doping level. This situation is consistent with the increase in $N\left(E_{\mathrm{F}}\right)$ upon doping, if $\beta$ is independent of the doping level. The observation that $T_{0}$ is larger in $\mathrm{Sr}_{1+x} \mathrm{La}_{1-x} \mathrm{CrO}_{4+\delta}\left(1.40-5.06 \times 10^{8} \mathrm{~K}\right)$ than that in $\mathrm{La}_{2} \mathrm{CuO}_{4}\left(0.3-7 \times 10^{6} \mathrm{~K}\right)$, ${ }^{3)}$ in which the conduction behavior has been reported insulating in the wide temperature range (room temperature to $2 \mathrm{~K}$ ), reflects a larger value of $\beta$ or a lower DOS at the Fermi level in the present system. It is reported that in $(\mathrm{La}, \mathrm{Sr})_{2} \mathrm{CuO}_{4}$ on the verge of the $\mathrm{MI}$ transition, $T_{0}$ is reported to be much smaller, $1-200 \mathrm{~K} .{ }^{2)}$ The density of states at the Fermi level in this study is expected to be large enough because the doping progressed to the order of several tens percents. Therefore, the larger $T_{0}$ in this system might be caused by the larger $\beta$, i.e., the localization length of the impurity states, $1 / \beta$, might be very small. It is considered that the smaller localization radius in $\mathrm{Sr}_{1+x} \mathrm{La}_{1-x} \mathrm{CrO}_{4+\delta}$ is not unusual, however, the larger value in the cuprate is anomalous. In the cuprate, copper ion has a $\mathrm{d}^{9}$ electronic configuration at an undoped and insulating state. This corresponds to the typical Jahn-Teller-ion, further the Jahn-Teller-distortion in the $\mathrm{Cu}-\mathrm{O}$ polyhedron is not cancelled upon hole-doping. ${ }^{14)}$ Therefore, the $\mathrm{Cu}-\mathrm{O}$ distance in the $a b$-plane is kept to be short unusually. It is supposed that this phenomenon causes the large overlap between $\mathrm{Cu} 3 \mathrm{~d}$ and $\mathrm{O} 2 \mathrm{p}$ states, so the doped-hole states spread over wide area in the lattice. In the present system, the length of $a$-axis, which corresponds to twice $\mathrm{Cr}-\mathrm{O}$ distance, decreases with $\mathrm{Sr}$ doping. Hence, the overlap between $\mathrm{Cr} 3 \mathrm{~d}$ and $\mathrm{O} 2 \mathrm{p}$ states may be enlarged upon doping. It is highly plausible that the decrease in $T_{0}$ upon doping is caused by the enlargement of the localization radius.

It is considered that the lack of the MI transition in this system may be due to the small localization radius of the impurity states, $1 / \beta$. We can not go further into this problem without added information. A speculative discussion is that the hybridization between $\mathrm{Cr} 3 \mathrm{~d}$ and $\mathrm{O} 2 \mathrm{p}$ characters in the hole-states is small. This situation will be elucidated by spectroscopic measurements such as photoemission and $\mathrm{X}$-ray absorption spectroscopies.

\subsection{Seebeck coefficient}

Seebeck coefficients in the VRH model can be expressed $^{7)}$ by

$$
S=\frac{1 k^{2}}{2 e}\left(T_{0} T\right)^{1 / 2}\left[\frac{\mathrm{d} \ln N(E)}{\mathrm{d} E}\right]_{E=E_{\mathrm{F}}}
$$

where $N(E)$ is the DOS at the energy of $E$. Therefore, it is expected that $S$ is proportional to $T_{0}{ }^{1 / 2}$ and $[\mathrm{d} \ln N(E) / \mathrm{d} E]_{E=E_{\mathrm{F}}}$ under the condition of constant temperature. Table 2 shows that observed Seebeck coefficient, $S(x)$, decreases with increasing doping level. The decrease in $S(x)$ upon doping seems to be consistent with the expectation from the Eq. (5), because $T_{0}(x)$, which was obtained independently from the electrical conductivity measurement, decreased with increasing a doping level. However, the decrease in $S(x)$ can not be explained by only decrease in $T_{0}(x)$, for example, the ratios $S(0.3) /$ $S(0)$ and $T_{0}{ }^{1 / 2}(0.3) / T_{0}{ }^{1 / 2}(0)$ were 0.26 and 0.53 , respectively. This suggests the decrease in $[\mathrm{d} \ln N$ $(E) / \mathrm{d} E]_{E=E_{\mathrm{F}}}$ upon doping. The term of $[\mathrm{d} \ln N$ $(E) / \mathrm{d} E]_{E=E_{\mathrm{F}}}$ expresses the variation rate of the DOS at the Fermi level. Because the DOS at the Fermi level increased upon the doping as described in the preceding subsection, the band width of the localized states may be increased upon doping. Consequently, it is speculated that the variation rate of the DOS at the Fermi level decreased with increasing doping level. On the other hand, one can see a good agreement between the ratios of $S(x) / S(0)$ and $T_{0}(x) / T_{0}(0)$ summarized in Table 2 . It is difficult to conclude whether this agreement is coincidental or not without added information.

\section{Summary}

Temperature dependence of the electrical conductivity and the Seebeck coefficient at room temperature of hole-doped $\mathrm{Sr}_{1+x} \mathrm{La}_{1-x} \mathrm{CrO}_{4+\delta}(0 \leq x \leq 0.3)$ were measured and analyzed based on the variablerange hopping (VRH) model. The results are summarized as follows:

(1) Linear relationship between $\ln \sigma$ and $T^{-1 / 4}$ or $T^{-1 / 3}$ was obtained. This shows that the mechanism of the electrical conduction in the materials was well described by three- or two-dimensional VRH model.

(2) The analysis of electrical conductivity based on three-dimensional VRH model showed the larger $T_{0}$ values, $10^{8} \mathrm{~K}$, in this system than that $0.3-7 \times 10^{6}$ $\mathrm{K}$ of an insulating $\mathrm{La}_{2} \mathrm{CuO}_{4}$. This suggests the localization length of impurity states in this system is much smaller than that of $\mathrm{La}_{2} \mathrm{CuO}_{4}$.

(3) The variation of Seebeck coefficients at room temperature suggests that not only the DOS but also the band width nearby the Fermi level were increased upon the hole-doping.

Acknowledgement This work was in part supported by the Science Research Promotion Fund from Japanese Private School Promotion Foundation and Science Research Fund from The Mechanical Industry Development and Assistance Foundation (MIDAF).

\section{References}

1) C. Quitman, D. Andrich, C. Jarchow, M. Fleuster, B. Beshoten, G. Guntherodt, V. V. Moshchalkov, G. Mante and R. Manzke, Phys. Rev., B46, 11813-25 (1992).

2) M. Z. Cieplak, S. Guha, H. Kojima, P. Lindenfeld, G. Xiao, J. Q. Xiao and C. L. Chien, Phys. Rev., B46, 5536-47 (1992).

3) M. A. Kastner, R. J. Birgeneau, C. Y. Chen, Y. M. Chiang, D. R. Gebbe, H. P. Jenssen, T. Junk, C. J. Peters, P. J. Pi- 
cone, T. Thio, T. R. Thurston and H. L. Tuller, Phys. Rev., B37, 111-17 (1988).

4) B. Fisher, G. Koren, J. Genossar, L. Patlagam and E. L. Gartstein, Physica C, 176, 75-79 (1991).

5) R. C. Budhani, S. H. Tzeng and R. F. Bunsheh, Phys. Rev., B36, 8873-76 (1987).

6) J. M. Valles, Jr., A. E. White, K. T. Short, R. C. Dynes, J. P. Garno, A. F. J. Levi, M. Anzlowar and K. Baldwin, Phys. Rev., B39, 11599-602 (1989).

7) N. F. Mott and E. A. Davis, "Electronic Processes in NonCrystalline Materials", 2nd ed., Oxford University Press, London (1979).

8) B. I. Shklovskii and A. L. Efros, "Electronic Properties of Doped Semiconductors", Springer-Verlag, Berlin (1984).
9) T. Omata, K. Ueda, N. Ueda, M. Katada, S. Fujitsu, T. Hashimoto and H. Kawazoe, Solid State Commun., 88, 80711 (1993).

10) T. Omata, K. Ueda, H. Hosono, M. Katada, N. Ueda and H. Kawazoe, Phys. Rev., B49, 10194-99 (1994).

11) T. Omata, K. Ueda, H. Hosono, T. Miyazaki, S. Hasegawa, N. Ueda and H. Kawazoe, Phys. Rev., B49, 10200-05 (1994).

12) T. Omata, H. Ikawa, S. Fujitsu, H. Hosono, N. Ueda and H. Kawazoe, Solid State Commun., in press.

13) G. Blasse, J. Inorg. Nucl. Chem., 27, 2683-84 (1965).

14) S. Kambe, K. Kishio, K. Kitazawa, K. Fueki, H. Takagi and S. Tanaka, Chem. Lett., 547-50 (1987). 